\title{
Pseudo-ensemble simulations and Gibbs-Duhem integrations for polymers
}

\author{
Fernando A. Escobedo and Juan J. de Pablo \\ Department of Chemical Engineering, University of Wisconsin-Madison, Madison, Wisconsin 53706-1691
}

(Received 3 September 1996; accepted 14 November 1996)

\begin{abstract}
Pseudo-ensemble simulations and Gibbs-Duhem integrations are formulated within the framework of the expanded grand canonical ensemble. Pseudo-isobaric-isothermal simulations are proposed in which volume moves are replaced by fluctuations in the number of molecular segments. For large systems of dense athermal polymers, this pseudo-isobaric-isothermal method is shown to achieve mechanical equilibration faster than both conventional volume moves and the recently proposed slab volume moves. Pseudo-ensembles are also discussed for Gibbs ensemble simulations and canonical simulation (of the chemical potential). It is shown that coexistence curves for pure homopolymers and polymer mixtures can be traced by performing a numerical integration of the Gibbs-Duhem equation based on (expanded) grand canonical simulations. The validity of the methods is demonstrated by tracing the vapor-liquid coexistence curve of pure square-well chains and the liquid-liquid binodal curve of a blend of square-well chains. (C) 1997 American Institute of Physics. [S0021-9606(97)51507-8]
\end{abstract}

\section{INTRODUCTION}

Molecular simulation of volumetric properties and phase equilibria of model fluids almost invariably requires sampling an ensemble in which the volume and/or the number of particles are allowed to fluctuate. Simulations in the isobaric-isothermal (NPT), grand canonical (GC), and Gibbs ensemble are relevant examples of techniques which involve such fluctuations and have become standard tools in the field of molecular simulation. ${ }^{1,2}$ In practice, implementing such fluctuations can be challenging, particularly for polymeric fluids; molecular insertion-deletion attempts become increasingly difficult as the chain length increases, and the effectiveness of volume moves deteriorates significantly as larger systems are simulated. Although several approaches have been proposed to overcome the difficulties associated with particle insertions (e.g., Refs. 1 and 3-7), it appears that a single, all-purpose approach is not available; instead, several specialized techniques will be required to broaden the spectrum of systems amenable to molecular simulation.

To overcome the transfer of particles required for the simulation of phase equilibria in the Gibbs ensemble, for example, Kofke ${ }^{5}$ proposed tracing the coexistence envelope of pure fluids by numerical integration of the Clapeyron equation. The initial conditions for the integration are provided by knowledge of one coexistence point. The method requires simultaneous isobaric-isothermal simulations of the two coexisting phases; unfortunately, for multicomponent mixtures the method cannot generally avoid particle insertions. ${ }^{8}$

Resorting to a similar combination of molecular simulation and numerical methods, Mehta and Kofke ${ }^{6}$ later proposed a pseudo-grand canonical (pseudo-GC) simulation technique in which particle insertions and deletions are replaced by volume fluctuations. In dense fluids the success rate of molecular insertion and deletion attempts can be prohibitively small; the pseudo-GC approach can therefore lead to faster convergence to the equilibrium density $(\rho)$ than con- ventional GC simulations (as shown in Ref. 6 for the hard sphere fluid). The pseudo-GC technique requires evaluation of the chemical potential $(\mu)$ and the pressure $(P)$ at the densities explored by the system. The approach has been extended to formulate a pseudo-Gibbs ensemble technique where particle transfers are replaced by concerted volume fluctuations. ${ }^{7}$ Although chemical potential evaluations in dense fluids are also difficult, the advantage of these pseudoensemble methods resides in their ability to accommodate alternative methods of accomplishing such evaluations.

The premise underlying the methods described above is the long-held tenet that molecular insertion and deletion attempts (or the exchange of particles) are more difficult to accomplish than volume fluctuations. While this is the case for many systems at high densities, the opposite is true for systems in which volume fluctuations are prohibitively sluggish. Slow convergence in volume sampling occurs for large systems at liquidlike densities, i.e., for simulation boxes containing several thousand sites. Figures of that magnitude are often encountered in the study of complex fluids (polymers, critical phenomena, electrostatic interactions, etc.). A complementary element that supports an approach that favors particle insertions over volume fluctuations is the recent development of expanded grand canonical (expanded GC) and expanded Gibbs ensembles, ${ }^{9}$ which have been shown to significantly facilitate molecular insertion-deletion or exchange attempts for polymers (even at high densities).

In this work we present a novel approach for the development of pseudo-ensemble simulation methods which is first contrasted to the original pseudo-GC prescription and then applied to formulate a pseudo- $N P T$ ensemble technique within the framework of the expanded GC ensemble. To demonstrate the usefulness of the pseudo-NPT method, we compare its performance to that of other methods (conventional volume moves ${ }^{1}$ and slab volume moves ${ }^{10}$ ) by monitoring the rate of mechanical equilibration for a system of hardcore 32-mers upon the application of a step change in pressure. Extensions of this approach are also discussed for 
Gibbs-type ensembles and for the canonical ensemble. A pseudo-Gibbs ensemble prescription is tested by generating a coexistence point for a square-well octamer system. Finally, we show how the Gibbs-Duhem equation can be integrated to trace coexistence envelopes for single and multicomponent systems in such a way that simultaneous (expanded) grand canonical simulations are performed on the individual coexisting phases. The technique is demonstrated by comparing select results of phase coexistence for chain systems to those obtained by means of other methods. Two cases are examined in this work: vapor-liquid equilibrium for an octamer fluid and isobaric liquid-liquid equilibrium for a binary mixture of octamers. In both examples, particles interact via a square-well potential energy function.

\section{PSEUDO-GRAND CANONICAL SIMULATIONS}

In a GC ensemble, the temperature and chemical potential of a system are specified and the density is evaluated by a sampling process that entails fluctuations of the number of particles at fixed volume. By exploiting the nature of the intensive variable associated with the Helmholtz free energy, Mehta and Kofke ${ }^{6}$ arrived at the following Metropolis-type acceptance criterion for volume changes $(\Delta V)$ designed to mimic particle insertion or deletion attempts:

$$
\min \left[1, \exp \left(\Gamma_{\mathrm{PGC}}\right)\right]
$$

where

$$
\begin{aligned}
\Gamma_{\mathrm{PGC}}= & \Delta N\left[\beta \mu_{\mathrm{GC}}-\beta \mu(\rho)+Z(\rho)\right]+N \ln \frac{V+\Delta V}{V} \\
& -\beta \Delta U
\end{aligned}
$$

and where

$$
\Delta N=-\Delta V \frac{N}{V+\Delta V} .
$$

In Eq. (1) $\mu_{\mathrm{GC}}$ is the prescribed grand canonical chemical potential, $V$ is the volume of the system, $\beta=1 / k_{B} T, N$ is the number of molecules in the system, and $\Delta U$ is the change in potential energy entailed by the volume change. To apply Eq. (1), the chemical potential $\mu(\rho)$ and compressibility factor $Z(\rho)$ must be known at the instantaneous density of the system $\rho$. To that end, methods to evaluate both $\mu$ and $P$ must also be implemented. Mehta and Kofke ${ }^{6}$ accumulated instantaneous values of $\mu$ and $P$ into density histograms; the values of $\mu(\rho)$ and $P(\rho)$ to be used in Eq. (1) were then obtained from (increasingly refined) empirical correlations fitted to histogram data. For the case of a hard sphere fluid, Mehta and Kofke used ghost particle insertions to evaluate $\mu(\rho)$ and the pressure equation to evaluate $Z(\rho){ }^{6}$

Subsequently, Camp and Allen ${ }^{7}$ pointed out that provided that the functions $\mu(\rho)$ and $Z(\rho)$ are accurately known, the limiting distribution probed by the pseudo-GC ensemble is that of the isothermal-isobaric (NPT) ensemble. This fact simply restates the dual nature of the problem at hand: The evaluation of the equilibrium density in a $\mu V T$ simulation could be replaced by an NPT simulation if the equilibrium pressure corresponding to the specified $\mu$ were known (and vice versa). By providing a good initial estimate of such a pressure, $P_{\text {eq }}$, and a scheme for its progressive refinement, an NPT simulation could therefore be used in lieu of a $\mu V T$ simulation. In that event, the Metropolis acceptance criterion for volume moves would be $\min \left[1, \exp \left(\Gamma_{N P T}\right)\right]$, where

$$
\Gamma_{N P T}=-\beta P_{\mathrm{eq}} \Delta V+N \ln \frac{V+\Delta V}{V}-\beta \Delta U .
$$

To find good estimates of $P_{\text {eq }}$, a boundary-value problem must be solved numerically for the following differential equation:

$$
d P=\rho d \mu \quad \text { constant } T .
$$

If the system is currently at some density $\rho$, we can write a Taylor expansion around the point $[\rho, \mu(\rho), P(\rho)]$ according to

$$
\begin{aligned}
P_{\mathrm{eq}}= & P(\rho)+\rho\left[\mu_{\mathrm{GC}}-\mu(\rho)\right]+\frac{\partial^{2} P}{\partial \mu^{2}}\left[\mu_{\mathrm{GC}}-\mu(\rho)\right]^{2} \\
& +\cdots,
\end{aligned}
$$

where identity (4) is implicit. If we truncate the expansion to first order and then update the calculation of $P_{\text {eq }}$ as the density of the system changes (such that the truncation becomes increasingly accurate as $\rho$ approaches its equilibrium value) we arrive at a prescription for a pseudo-GC simulation. It turns out that such a scheme is precisely that of Mehta and Kofke; by substituting the estimate of $P_{\text {eq }}$ and the definition of $\Delta N$ [Eq. (2)] into Eq. (3), we arrive at Eq. (1).

A possible advantage of the interpretation given here (besides its simplicity) is that other, perhaps faster schemes for a pseudo-GC simulation could be formulated depending on how $P_{\text {eq }}$ is estimated and updated. Such freedom is not surprising (or detrimental) because for any practical implementation of a pseudo-ensemble method the overall simulation process will not be strictly Markovian. As an example, an alternative pseudo-GC implementation can proceed in predictor-corrector steps as follows:

(1) At the initial density of the system $\rho_{0}$, compute $\mu_{0}$ and $P_{0}$ by using any suitable method.

(2) Compute $P_{\text {eq }}$ from Eq. (5) truncated to first order (setting $\rho=\rho_{0}$, predictor step).

(3) Conduct conventional NPT volume fluctuations in accordance with Eq. (3) and update the current value of pressure according to (corrector steps)

$$
P_{\text {eq }}=P_{0}+\frac{1}{2}\left(\rho+\rho_{0}\right)\left[\mu_{\mathrm{GC}}-\mu_{0}\right] .
$$

(4) Once convergence to a certain density $\rho_{1}$ and $P_{\text {eq }}=P_{1}$ has occurred (as determined by some suitable criteria), set $\rho_{1}=\rho_{0}, P_{0}=P_{1}$, compute the chemical potential at the current density, and repeat the process from step (2) until global convergence of density is attained.

Step (3) employs a two-point implicit (trapezoid) formula for integrating Eq. (4), chosen here for its simplicity and stability. If the initial state is very different from the equilibrium 
state, the scheme outlined above can suffer from convergence problems; in such a case, one could aim at $\mu_{\mathrm{GC}}$ in stages. Variations on the same theme could also be obtained by using higher order integration schemes, such as the addition of the second-order term in Eq. (5), etc.

A possible advantage of the scheme outlined in this section over that proposed by Mehta and Kofke is that it reduces the number of chemical potential evaluations (these are only performed at a few density points, and not at every density point as in Mehta and Kofke's scheme). Also, it practically suppresses the need for pressure evaluations since only the initial point is required. In practice, however, such a gain will be of little significance since the convergence of pressure is much less troublesome than that of chemical potential. Of course, direct chemical potential simulation can always be replaced by extensive pressure evaluations by resorting to thermodynamic integration of Eq. (4), i.e., $\beta \mu$ $=\beta \mu_{0}+Z-Z_{0}+\int_{\rho_{0}}^{\rho}\left(\beta P / \rho^{2}\right) d \rho$.

\section{EXPANDED GRAND CANONICAL ENSEMBLE}

A complete account of this method can be found in the original source ${ }^{9}$ for completeness, we provide here a brief summary. For polyatomic molecules, the system (at any given time) consists of $N$ full molecules of length $n$ and one tagged molecule of length $n_{y}$ (the total number of molecules is $N_{y}$ ). The size or length of the tagged molecule varies according to its current state $y$. It can eventually disappear $(y=1)$ or become a full molecule $(y=M=$ number of specified states); in either case, another molecule (existing or new) becomes tagged. Segmental (or multisegmental) molecular creations $(\Delta=+1)$ and deletions $(\Delta=-1)$ of the tagged chain are attempted once every $N_{\text {state }}$ molecular moves and accepted according to $\min \left[1, \exp \left(\Gamma_{\mathrm{EGC}}\right)\right]$, where

$$
\Gamma_{\mathrm{EGC}}=\Delta \ln R_{W}+\Psi_{y+\Delta}-\Psi_{y}
$$

and where the $\Psi$ 's are preweighting factors given by

$$
\Psi_{y}=\omega_{y}\left[\beta \mu_{\mathrm{eq}}-\ln \left(\frac{N_{y}}{V}\right)\right] .
$$

In Eq. (7), $\omega_{y}$ is a fractional weight such that $\omega_{1}=0, \omega_{M}=1$, and intermediate values are chosen such that all states of the tagged chain are visited with approximately the same frequency. ${ }^{9}$ In Eq. (6), $R_{W}$ is the Rosenbluth weight associated with a biased segmental growth process; for flexible molecules $R_{W}$ is given by

$$
R_{W}=\prod_{i=n_{A}}^{n_{B}} N_{\mathrm{sp}}^{-1}(i) \sum_{j=1}^{N_{\mathrm{sp}}(i)} \exp \left[-\beta U_{j}(i)\right],
$$

where $n_{A}=\min \left(n_{y}, n_{y+\Delta}\right)+1 \quad$ and $n_{B}=\max \left(n_{y}, n_{y+\Delta}\right)$, $N_{\text {sp }}(i)$ is the number of random trial positions for the $i$ th segment of the molecule, and $U_{j}(i)$ is the interaction energy between the $i$ th segment and the system. Statistics on the system's density are collected when the tagged chain visits the end states.

The extension of this approach to multicomponent systems is straightforward; independent transition attempts for each species are accepted according to Eq. (6) provided that the $\Psi$ 's, $\mu_{\text {eq }}$, and $N_{y}$ correspond to the relevant species. It is finally noted that the conventional grand canonical prescription for pure fluids can be recovered from Eqs. (6)-(8) by setting $M=2$ states and $N_{\mathrm{sp}}=1$.

\section{PSEUDO-NPT SIMULATIONS}

\section{A. Methodological aspects}

In a conventional NPT simulation, the equilibrium density of the system is attained by fluctuations of its volume at constant number of molecules (for fixed $P$ and $T$ ). In a pseudo-NPT ensemble, $P$ and $T$ are also specified but trial volume moves are replaced by molecular insertion or deletion attempts (at fixed volume). The line of arguments given by Mehta and Kofke to arrive at a prescription for pseudo-GC moves can also be followed to derive acceptance criteria for pseudo-NPT moves. However, identical results can be obtained more directly by resorting to the alternative interpretation given in Sec. II; we illustrate the implementation of pseudo-NPT simulations within the framework of the expanded GC ensemble of Sec. III. Equation (6) requires a prescription for estimating the chemical potential $\mu_{\mathrm{eq}}$ corresponding to the specified pressure $P_{\text {eq }}$ (and temperature). For concreteness, we adopt here a trapezoid predictor-corrector scheme

$$
\beta \mu_{\mathrm{eq}}=\beta \mu_{0}+\frac{1}{2}\left(\frac{1}{\rho}+\frac{1}{\rho_{0}}\right)\left(\beta P_{\mathrm{eq}}-\beta P_{0}\right),
$$

where $\rho$ is the current value of the molecular number density and the subindex ' 0 "' denotes a reference state (for the predictor step $\rho=\rho_{0}$ ). The simulation then proceeds as follows:

(1) At the outset of the simulation, evaluate $\mu_{0}$ and $P_{0}$ at the initial density of the system $\rho_{0}$. If $P_{0}$ differs substantially from the input pressure $P_{\text {eq }}$, aim first at an intermediate pressure $P_{\mathrm{eq}}^{\prime}=P_{0}+\gamma\left(P_{\mathrm{eq}}-P_{0}\right)$, where $0<\gamma<1$.

(2) Compute $\beta \mu_{\text {eq }}$ from Eq. (9).

(3) Conduct conventional expanded GC moves according to Eq. (6) and update the current value of $\beta \mu_{\mathrm{eq}}$ using Eq. (9), where $\rho$ is the "current block-average" density of the system.

(4) Once the system has converged to a density $\rho_{1}$ consistent with a value of $\beta \mu_{\mathrm{eq}}=\beta \mu_{1} \approx$ constant, set $\rho_{0}=\rho_{1}$, $\beta \mu_{0}=\beta \mu_{1}$, and recompute the pressure $P_{0}$.

(5) Iterate from step (3) until global convergence of the density is attained.

This scheme is designed to reduce the number of pressure evaluations (particularly far from the equilibrium density) and, more importantly, to avoid the often troublesome evaluation of the chemical potential (only the initial value is needed), $\mu_{\mathrm{eq}}$ is indirectly determined by exploiting the fast convergence of expanded GC moves. Furthermore, if a previous state is known it can be chosen as an initial point for the simulation, thereby eliminating the need for step (1). Also note that if the value of $P_{0}$ calculated initially is very different from $P_{\text {eq }}$, a rough estimate of $\mu_{0}$ is unlikely to have a significant effect on the overall convergence of the method. 
To dampen fluctuations of the $\beta \mu_{\mathrm{eq}}$ estimates from Eq. (9), current "block averages" of $\rho$ are used in step (3), rather than instantaneous values. A block average is obtained from a presized set of recent evaluations (e.g., after a preset number of cycles of expanded GC moves or after the last pressure update).

The criteria for determining if partial [step (4)] or overall [step (5)] convergence has occurred is based on histograms of $\rho, P_{0}$, and $\mu_{\mathrm{eq}}$ : empirical fits, as employed by Mehta and Kofke, ${ }^{6}$ could also be used but have not been implemented here. Of course, once updates of $\mu_{\mathrm{eq}}$ have ceased, the simulation process becomes Markovian.

\section{B. Application and efficiency of pseudo-NPT simulations}

To illustrate the implementation of our pseudo- $N P T$ prescription, in this section we examine the relaxation of a polymer melt to equilibrium after a step increase of the imposed pressure. We simulate a homopolymer fluid of freely jointed chains consisting of 32 hard spheres each. Even for such a modest chain length, a relatively large system would be required to minimize finite size effects on certain properties of interest, such as site-site distribution functions, chain dimensions, and chemical potential. At a site density of 0.7 [or polymer packing fraction $\eta=0.367]$ a cubic simulation box of edge $=32$ bead diameters $(\sigma)$ would result in a system with $N_{s} \approx 23000$ beads. For this particular molecular model, however, we could probably get away with a simulation box having an edge of just $16 \sigma$ (i.e., $N_{s} \sim 2900$ beads), because chain conformations rarely extend beyond that distance. This would not apply, however, to less flexible molecules, longer range interactions, etc. If we bear in mind that many aspects of polymer physics require simulation of coarse-grained molecular models having $>O\left(10^{2}\right)$ segments, it is easy to appreciate the need for efficient simulation methods for systems of $O\left(10^{4}\right)$ sites.

Based on these considerations, we have chosen to study two system sizes: (i) one with $N \sim 150,32$-mers and (ii) another containing $N \sim 300,32$-mers. Three methods are considered for isobaric simulations.

(1) Conventional volume moves, ${ }^{11,1}$ which entail isotropic changes of the simulation box size; the centers of mass of all molecules are rescaled while the intramolecular configurations remain unchanged. One volume move is attempted every $0.24 N_{s}$ molecular move attempts.

(2) Slab volume moves,${ }^{10}$ where the thickness of a randomly located slab of the simulation box (thickness $\sim \sigma$ ) is increased or decreased; molecular portions contained within the slab are rearranged to preserve chain connectivity, but the rest of the system remains unchanged. One slab volume move is attempted every $0.08 N_{s}$ molecular move attempts.

(3) Pseudo-NPT volume moves, as described in the previous section. Segmental creation or deletions $\left(\Delta n_{y}=n_{y+1}\right.$ $\left.-n_{y}=1, \omega_{y}=n_{y} / n\right)$ are attempted every $N_{\text {state }} \sim \frac{2}{3} N$ trial molecular moves $\left(N_{\mathrm{sp}}=40\right)$.

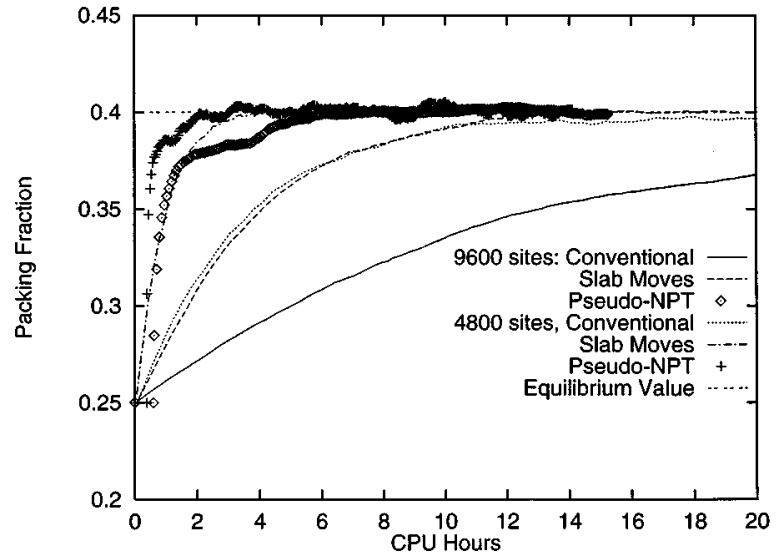

FIG. 1. Time evolution of the packing fraction of a 32-mer hard-chain system upon application of a step increase in pressure. The equilibrium density corresponding to the imposed pressure $\left(P^{*}=3.17\right)$ is $\eta=0.400$. Results are presented for two system sizes, one containing about 300 molecules (9600 sites) and the other 150 molecules (4800 sites).

Extended continuum configurational-bias moves, ${ }^{12}$ reptation, and translation moves were used for molecular relaxations using identical parameter settings for the three methods. For pseudo-NPT simulations, we adopted Nezbeda's pressure equation as implemented by Chang and Sandler ${ }^{13}$ for evaluating the pressure of the system; $0.5 N_{s}$ snapshots were employed for each evaluation.

The efficiency test performed in this section is similar to that originally reported in Ref. 10; a pre-equilibrated system at a packing fraction $(\eta)$ of 0.25 (consistent with a pressure of $\left.P^{*}=\beta P \sigma^{3}=0.56\right)$ is suddenly subjected to a pressure of $P^{*}=3.17$ (corresponding to an equilibrium packing fraction of $0.4^{14}$ ). The range of densities covered is representative of liquidlike conditions. The evolution of the density toward equilibrium is monitored as a function of CPU time on a 266 $\mathrm{MHz}$ Alpha-processor workstation.

The results are shown in Fig. 1. It is first noted that, regardless of the method, the convergence to equilibrium slows down with increasing system size. For conventional volume moves, as the number of sites in the system increases so does the likelihood of finding two sites positioned so close to each other that even a small volume fluctuation will result in an overlap. Therefore, to achieve a certain fraction of successful trial volume moves (e.g., 40\%), changes must necessarily be smaller. This attrition is magnified as the density increases. Slab volume moves face a similar problem but to a lesser extent, by sampling only a subregion of space, the slab algorithm can find some slabs where molecular portions have configurations favorable for a volume change. It should be pointed out that both methods employ a simulation box partitioned into cubic cells small enough to accommodate at most one site. Such a computational artifact reduces significantly the task of locating neighboring sites required to evaluate the system's energy after a volume move; it leads to a practically ideal linear scaling of the number of computations with the number of sites in the system. In both cases, however, the attrition effects mentioned above give rise to a 
computational performance that scales as $\propto N_{s}^{\nu}$ with $\nu>1$ (the specific value of $\nu$ depends on the density, see Fig. 1). For example at $\eta=0.35$ the slope of the curve for the small system is about three times that of the larger system. Although additional special tricks could be implemented to alleviate atrittion, these are not generally applicable. For the pseudoNPT methods, however, the slowing down simply reflects the fact that for a given density increase one needs to insert twice as many molecules if the system is twice as large, i.e., computer time scales exactly as $\propto N_{s}$.

The initial value of $\beta \mu_{0}$ needed for the pseudo-NPT simulations was evaluated by the expanded canonical method, ${ }^{15}$ which can be regarded as a special case of the expanded GC implementation (thus sharing the same basic code). The first flat portion of the curves in Fig. 1 (from 0 to $\sim 1 / 2 \mathrm{~h}$ ) corresponds to such evaluations. It is noted that since a rough estimate is sufficient here, one could evaluate $\mu_{0}$ based on information from the insertion of a short chain (using configurational bias $^{3}$ ) and from that of a single segment (by invoking Kumar et al.'s method ${ }^{4}$ in the long chain limit). Alternatively, the initial value of $\beta \mu_{0}$ could be obtained from a short pseudo-canonical simulation as described later in this paper. A value of $\gamma=0.75$ is used (Sec. IV A) because for the initial state $P_{0} \sim 0.56 \ll P_{\text {eq }}=3.17$. This leads to an initial underestimation of $\mu_{\mathrm{eq}}$ and $\rho$ that shows up in the (pseudo-NPT) curves of Fig. 1 in the form of plateau regions around $\eta \sim 0.38$. After a pressure evaluation, local convergence of density was deemed satisfactory when 200 entries of "running density averages" were accumulated in one histogram bin of width $\Delta \eta=0.0013$. This was done to provide enough time for expanded GC moves to "substantially" converge to an equilibrium density. From the first plateau at $\eta \sim 0.38$ in Fig. 1, the averaging period alone takes over $1 / 2$ $\mathrm{h}$ in the small system and over $1 \mathrm{~h}$ in the large system. If too short a period were prescribed, convergence (if attainable) would become highly oscillatory because local equilibration is never achieved. Conversely, if too long a period were prescribed, the overall convergence could be delayed since the system would dwell for long periods of time in pseudoequilibrium states (particularly at the beginning of the simulation).

It is observed from Fig. 1 that pseudo-NPT simulations are more efficient than slab volume moves, which are in turn faster than conventional volume moves. These results, however, should be regarded as representative since they depend on our particular choice of simulation parameters (in particular, the frequency of volume move attempts was shown to be very important in Ref. 10; preliminary tests indicate that our settings are sensible choices although they may not be optimal). Parameter settings for pseudo-NPT simulations (discussed in the previous paragraph) are based on our limited experience. Pseudo-NPT and slab volume moves have comparable efficiency for the small system. For the large system, the pseudo-NPT method is about twice as fast as the slab method. This is due to the favorable scaling of pseudo-NPT calculations with system size. We also expect the performance of slab volume moves to deteriorate somewhat faster than that of the other methods for systems with long-range intersite interactions and for rigid molecules, because in such cases the slab thickness must be larger. Pseudo-NPT simulations are at least an order of magnitude faster than conventional volume moves in equilibrating either system. In fact, for both systems the conventional method failed to reach the equilibrium density during the total test time $(20 \mathrm{~h})$. An additional advantage of pseudo-NPT simulations over existing constant-pressure techniques is that they lead to more natural, efficient implementations on parallel computer architectures.

\section{Extension to the Gibbs ensemble}

In a Gibbs ensemble, equilibrium between two simulation boxes is attained by rearrangement of molecules, transfer of volume, and transfer of particles between the boxes. ${ }^{2,17}$ Camp and Allen ${ }^{7}$ have recently extended the pseudo-GC ensemble method to the Gibbs ensemble formalism. In a pseudo-Gibbs ensemble simulation, particle transfer moves are never attempted; instead, volume moves are conducted in such a way as to mimic the effect of such transfers. These pseudo exchange attempts of $\Delta N$ particles from one box (say, phase "II") to the other (phase "I") are accepted according to $\min \left[1, \exp \left(\Gamma_{\text {exch }}\right)\right]$, where

$$
\begin{aligned}
\Gamma_{\text {exch }}= & \Delta N\left[Z_{0}^{\mathrm{I}}-Z_{0}^{\mathrm{II}}-\beta\left(\mu_{0}^{\mathrm{I}}-\mu_{0}^{\mathrm{II}}\right)\right]+N^{\mathrm{I}} \ln \frac{V^{\mathrm{I}}+\Delta V^{\mathrm{I}}}{V^{\mathrm{I}}} \\
& +N^{\mathrm{II}} \ln \frac{V^{\mathrm{II}}+\Delta V^{\mathrm{II}}}{V^{\mathrm{I}}}+\beta\left(\Delta U^{\mathrm{I}}+\Delta U^{\mathrm{II}}\right) .
\end{aligned}
$$

In Eq. (10), the subindex " 0 " denotes the reference state. For this application, the reference state is that corresponding to the trial density (after particle exchange). Volume increments are related to $\Delta N$ as follows:

$$
\Delta V^{\mathrm{I}}=-\frac{V^{\mathrm{I}} \Delta N}{N^{\mathrm{I}}+\Delta N}, \quad \Delta V^{\mathrm{II}}=+\frac{V^{\mathrm{II}} \Delta N}{N^{\mathrm{II}}+\Delta N} .
$$

The above criterion for pseudo-transfer moves was derived by resorting to the partition function of the Gibbs ensemble and the properties of the free energy density. ${ }^{7}$ As in previous sections, it is instructive to outline an alternative derivation. If, to first order, we write $\mu_{\mathrm{eq}}=\mu_{0}+\rho_{0}^{-1}\left(P_{\mathrm{eq}}-P_{0}\right)$ for each phase and assume that (near coexistence) the values of $P_{\text {eq }}$ and $\mu_{\text {eq }}$ are the same in both phases, we can solve for $P_{\text {eq }}$ to arrive at

$$
\beta P_{\mathrm{eq}}=\frac{1}{v_{0}^{\mathrm{I}}-v_{0}^{\mathrm{II}}}\left[\beta \mu_{0}^{\mathrm{II}}-\beta \mu_{0}^{\mathrm{I}}-Z_{0}^{\mathrm{II}}+Z_{0}^{\mathrm{I}}\right],
$$

where $v_{0}=\rho_{0}^{-1}$. If we now propose a volume change $\Delta V^{J}$ for phase $J$, the factor $\Gamma_{N P T}$ of acceptance criterion (3) becomes

$$
\Gamma_{N P T}^{J}=-\beta P_{\mathrm{eq}} \Delta V^{J} N^{J} \ln \frac{V^{J}+\Delta V^{J}}{V^{J}}-\beta \Delta U^{J}, \quad J=\mathrm{I}, \mathrm{II} .
$$


Correlated volume changes in both phases (that mimic particle transfers) are then accepted with probability $\min \left[1, \Gamma_{N P T}^{\mathrm{I}, \mathrm{II}}\right]$, where $\Gamma_{N P T}^{\mathrm{I}, \mathrm{II}}=\Gamma_{N P T}^{\mathrm{I}} \Gamma_{N P T}^{\mathrm{II}}$. The identity $\Gamma_{N P T}^{\mathrm{I}, \mathrm{II}}=\Gamma_{\text {exch }}$ follows from Eqs. (11)-(13).

Our derivation suggests that, at least in principle, pseudo-transfer moves need not be coupled. If Eq. (12) does converge to the equilibrium pressure $P_{\text {eq }}$ (e.g., $\left.v_{0}^{\mathrm{I}} \neq v_{0}^{\mathrm{II}}\right)$, the use of criteria (13) implies that each phase is simulated within the framework of an NPT-type of ensemble. Such implementation would correspond to a method that conducts iterations of the $N P T-\mu$ method $^{18}$ in a single run. Of course, the coupling of pseudo-transfer moves can improve the rate of convergence and stability of the simulation.

We now formulate a pseudo-Gibbs ensemble method in which volume moves are suppressed in favor of particle exchanges. For concreteness, we adopt a trapezoid predictorcorrector formula for the pressure, $P_{\text {eq }}=P_{0}+\widetilde{\rho}\left(\mu_{\mathrm{eq}}-\mu_{0}\right)$ (where $\tilde{\rho}$ is the average molecular density between the reference and current states). If such a formula is written for each phase and the equality of pressures is enforced, we arrive at the following expression for the chemical potential:

$$
\beta \mu_{\mathrm{eq}}=\frac{1}{\widetilde{\rho}^{\mathrm{I}}-\widetilde{\rho}^{\mathrm{II}}}\left[\beta P_{0}^{\mathrm{II}}-\beta P_{0}^{\mathrm{I}}-\widetilde{\rho}^{\mathrm{II}} \beta \mu_{0}^{\mathrm{II}}+\tilde{\rho}^{\mathrm{I}} \beta \mu_{0}^{\mathrm{I}}\right] .
$$

Equation (14) can be employed to perform coupled grand canonical moves in each box so as to mimic a transfer of volume between the regions [in analogy to the strategy of Eqs. (10) and (11)]. While such an approach may offer some advantages, we restrict the remaining discussion to a simpler implementation in which grand canonical (or expanded GC) moves are performed independently in each phase. Equation (14) provides the value of the chemical potential required in the acceptance criteria for such moves [e.g., Eq. (6)]. In principle, the simulation entails three types of moves: (i) molecular rearrangements (in each box), (ii) conventional molecular transfer moves between the phases; for polymers, this can be accomplished via configurational-bias or expanded Gibbs ensemble moves, and (iii) pseudo-volume moves, performed via (expanded) grand canonical moves. If molecular transfer moves are efficient, pseudo-volume moves could be attempted after sufficiently long intervals so as to ensure the equality of chemical potentials in both phases $\left(\mu_{0}^{\mathrm{I}}=\mu_{0}^{\mathrm{II}}=\mu_{0}\right)$; in such a case, Eq. (14) reduces to

$$
\beta \mu_{\mathrm{eq}}=\beta \mu_{0}-\frac{\beta P_{0}^{\mathrm{I}}-\beta P_{0}^{\mathrm{II}}}{\widetilde{\rho}^{\mathrm{I}}-\widetilde{\rho}^{\mathrm{II}}} .
$$

Pseudo-Gibbs ensemble techniques (based on either NPT or GC simulations) that resort to decoupled moves are remminiscent of thermodynamic integration.

An expanded Gibbs ensemble ${ }^{9}$ differs from a conventional Gibbs ensemble in that chemical potential equilibration is achieved by "segmental" exchanges, i.e., by concerted segmental insertions onto a tagged chain in one phase accompanied by deletion of segments from a complement tagged chain in the other phase. The only additional consideration pertaining to a pseudo-expanded Gibbs ensemble (beyond the guidelines for a pseudo-Gibbs ensemble) is that the usual correlation between states of the tagged chains in each phase must be relaxed ${ }^{9}$ to accommodate the segmental insertion-deletion attempts required by pseudo-volume moves.

There are several ways of implementing pseudo-Gibbs ensemble simulations based on Eq. (14). As an example, we formulate a variant that does not rely on particle exchange moves, i.e., it operates by conducting two simultaneous grand canonical simulations (one for each phase), the only coupling between them is through the prescribed chemical potential. Further, this version does not require chemical potential evaluations. To simulate the coexistence properties of a system at a prescribed temperature, the following steps are taken [see also Fig. 2(a)]:

(1) Perform short preliminary simulations (on a small system) to quickly locate two state points I and II, lying on the stable region of each phase. Expanded GC simulations can rapidly show whether the state of the system is liquid or vapor for a prescribed chemical potential. This task can of course be facilitated if another point on the coexistence curve is already known.

(2) Perform expanded GC simulations at states I and II (i.e., at $\mu_{0}^{\mathrm{I}}$ and $\mu_{0}^{\mathrm{II}}$ ) to obtain accurate values of $\rho_{0}$ and $P_{0}$.

(3) Perform a pseudo-Gibbs ensemble simulation starting at conditions consistent with states I and II. Working chemical potentials for each phase are updated according to

$$
\beta \mu^{J}=\gamma \beta \mu_{0}^{J}+(1-\gamma) \beta \mu_{\mathrm{eq}}, \quad 0<\gamma<1, \quad J=\mathrm{I}, \mathrm{II},
$$

where $\mu_{\mathrm{eq}}$ is computed from Eq. (14). The simulation proceeds by a sequence of equilibration and averaging periods. An equilibration period, which follows an update of the chemical potential, allows the system to relax in response to the new conditions (a refinement of $\mu_{\mathrm{eq}}$ is also possible). During the subsequent averaging period, statistics for $\rho$ and $P$ are collected; their average values are used at the end of this period to update reference values of $P_{0}$ and $\rho_{0}$ for each phase. Subsequently, $\mu_{\mathrm{eq}}, \mu^{\mathrm{I}}$, and $\mu^{\mathrm{II}}$ are computed from Eqs. (14) and (15) and a new equilibration period can then proceed. Convergence to coexistence conditions occurs when $\mu^{\mathrm{I}}=\mu^{\mathrm{II}}\left(\right.$ and $\left.P^{\mathrm{I}}=P^{\mathrm{II}}\right)$.

Note that the first two steps could also be accomplished via NPT simulations. The mixing of the old and new solutions for the chemical potential [Eq. (15)] is important because the extrapolated value of $\mu_{\text {eq }}$ from Eq. (14) could be well within the single-phase domain, thereby leading to phase reversal in one of the simulation boxes.

We have tested this prescription by determining a vapor-liquid coexistence point for an octamer system with sites having a diameter equal to the bond length $=\sigma$ and interacting through a square-well potential energy function of well width $0.5 \sigma$ and well depth $\epsilon$. The pressure of the system, required for Eq. (14), can be determined with the pressure equation of Nezbeda as implemented by Tavares et al. ${ }^{16}$ Such an equation provides accurate results for the gas-phase pressure but not for the liquid phase, particularly close to 
coexistence where slow, large fluctuations prevail. This problem can be circumvented by evaluating the liquid-phase pressure indirectly by numerical integration of either the $\mu$ route,

$$
P=P_{0}+\int_{\mu_{0}}^{\mu} \rho d \mu^{\prime}
$$

or the $\kappa_{T}$ route,

$$
P=P_{0}+\int_{\rho_{0}}^{\rho}\left(\rho \kappa_{T}\right)^{-1} d \rho,
$$

where the subscript " 0 " denotes properties evaluated at the initial state (where the pressure was obtained accurately) and $\kappa_{T}=-(1 / v)(\partial v / \partial P)_{T}$ is the isothermal compressibility. The integration can be performed by fitting to a suitable functional form (e.g., a polynomial) the data points already generated in the simulation, i.e., to a set of points $\left[\rho^{J}, \mu^{J}\right]$ for the $\mu$ route, and a set of points $\left[\kappa_{T}^{J}, \rho^{J}\right]$ for the $\kappa_{T}$ route. The fitted function can then be integrated analytically. While the $\mu$ route is more generally applicable and naturally suited to our method, we report data obtained from the $\kappa_{T}$ route only, coexistence properties obtained by both routes were statistically indistinguishable. The isothermal compressibility can be effortlessly evaluated during a grand canonical-type simulation (or the expanded GC averaging periods of the proposed pseudo-Gibbs method) from ${ }^{1}$

$$
\kappa_{T}=\beta V\left[\frac{\left\langle\rho^{2}\right\rangle}{\langle\rho\rangle^{2}}-1\right] .
$$

Simulated values of $\kappa_{T}$ exhibited sufficiently small uncertainties to permit reliable calculations of the liquid phase pressure. Our simulations were conducted at $T^{*}=k_{B} T /$ $\epsilon=1.9$; about 130 molecules were present in each simulation box. Each averaging period consisted of $3 \times$ equilibration period $=5 \times 10^{6}$ elementary molecular move attempts. A conservative value of $\gamma=0.75$ was used in Eq. (15) (note that a smaller value could be used in this case, thereby leading to faster convergence). The initial $\left(\beta \mu, P^{*}, \eta\right)$ points were $(-6.0,0.0274,0.3013)$ for the liquid phase, and $(-7.0$,

TABLE I. Results of Gibbs-Duhem integration for the vapor-liquid coexistence $\left(\eta_{V}-\eta_{L}\right)$ of a homopolymer fluid of square-well chains of eight sites. Results for the starting point at $T^{*}=1.9$ were obtained from a Gibbs ensemble simulation.

\begin{tabular}{lccc}
\hline \hline \multicolumn{1}{c}{$\beta$} & $T^{*}$ & $\eta_{L}$ & $\eta_{V}$ \\
\hline 0.58824 & 1.7000 & 0.333 & 0.0023 \\
0.570 & 1.7544 & 0.323 & 0.0037 \\
0.555 & 1.8018 & 0.313 & 0.0055 \\
0.543 & 1.8416 & 0.303 & 0.0077 \\
0.534 & 1.8727 & 0.294 & 0.0100 \\
0.52632 & 1.9000 & 0.286 & 0.0125 \\
0.520 & 1.9231 & 0.277 & 0.0153 \\
0.515 & 1.9418 & 0.271 & 0.0180 \\
0.511 & 1.9570 & 0.265 & 0.0200 \\
0.507 & 1.9724 & 0.257 & 0.0235 \\
0.503 & 1.9881 & 0.249 & 0.0270 \\
0.500 & 2.0000 & 0.244 & 0.0300 \\
\hline \hline
\end{tabular}

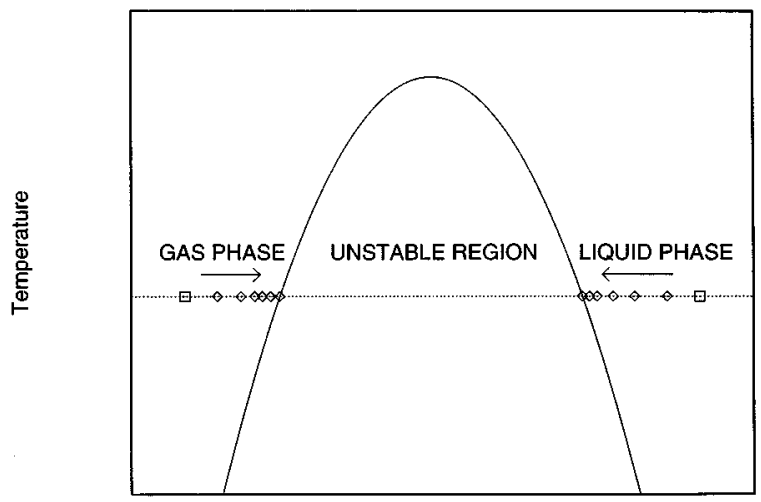

(a)

Density

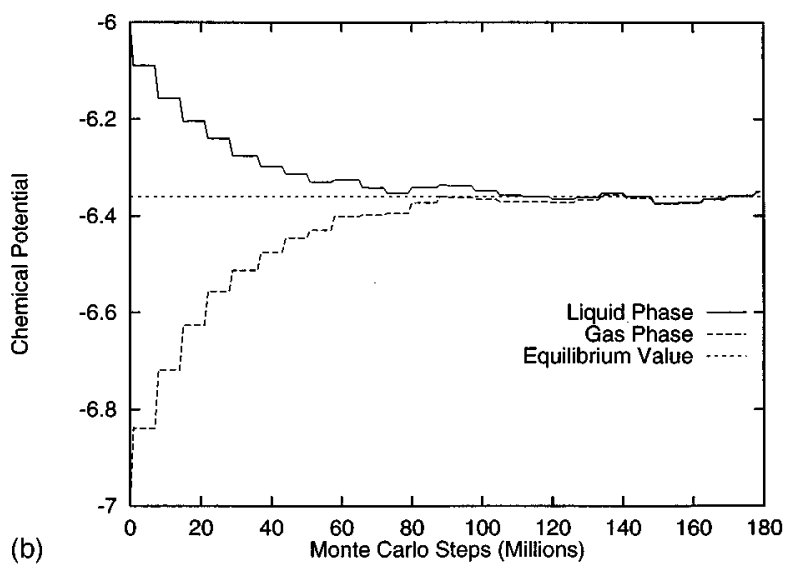

FIG. 2. Convergence of a proposed pseudo-Gibbs ensemble simulation method toward coexistence conditions at a given temperature, (a) schematic representation on the $T-\rho$ plane showing the coexistence curve (full line), the initial simulated states (squares) and the sequence of points generated throughout a pseudo-Gibbs simulation (diamonds). (b) Evolution of expanded GC chemical potential for the liquid phase (upper curve) and the gas phase (lower curve) of a square-well octamer fluid at $T^{*}=1.9$. The updates of chemical potential occur after $\sim 7 \times 10^{6}$ elementary Monte Carlo steps and are governed by Eqs. (14) and (15).

$0.00187,0.00496)$ for the vapor phase. The coexistence point found from conventional Gibbs ensemble simulation (see Table I) is $\beta \mu=-6.36, P^{*}=0.0023, \eta_{L}=0.286$, and $\eta_{V}=0.00125$. Identical results (within the uncertainty of the simulations) were obtained from our pseudo-Gibbs prescription; Figure 2(b) shows the evolution of the chemical potentials $\mu^{\mathrm{I}}$ and $\mu^{\mathrm{II}}$ throughout the course of the simulation. Note that, at the conditions studied here, the interfacial free energy is large enough to prevent phase reversal for small departures around the equilibrium chemical potential.

Independent pseudo-volume moves are only applicable if $\rho^{\mathrm{I}} \neq \rho^{\mathrm{II}}$. This implies that some care must be exercised in planning and monitoring the simulation. For example, the simulation should not be started from two phases with identical densities; preferably, the initial densities should be as different from each other as sensibly possible. Also, the method is likely to fail near the critical point.

The implementation of pseudo-Gibbs ensemble simula- 
tions for multicomponent systems will be addressed in a forthcoming publication.

\section{PSEUDO-CANONICAL SIMULATION OF THE CHEMICAL POTENTIAL}

The task of evaluating the relationship between $\rho$ and $\mu$ for a pure fluid at some discrete points (and at a preset temperature) can be undertaken either by simulations in a canonical $(N V T)$ ensemble or in a GC $(\mu V T)$ ensemble. From a computational point of view, a canonical ensemble is often preferred for simple fluids because reliable alternative methods are now available for simulation of the chemical potential, even at difficult conditions. For complex fluids such as polymers, however, the methods available for accurate evaluation of the chemical potential in a canonical ensemble are more limited.

A method that possesses wide applicability for polymers (i.e., it is not restricted to short chains or simple molecular structures) is the expanded canonical method, ${ }^{15}$ which gradually couples and decouples a tagged chain to a system by resorting to a segmental process analogous to that encountered in the expanded GC ensemble described in Sec. III. In practice, it turns out that the expanded canonical method requires more preliminary work (to estimate preweighting factors) and is invariably more time consuming than the expanded GC simulation for comparable conditions (i.e., same average density, temperature, and system size). This suggests that it would be advantageous to formulate a pseudocanonical ensemble method for simulation of the chemical potential (at a preset system density) that takes advantage of the fast convergence of expanded GC moves. To implement such a method, we require a prescription that generates progressively refined estimates of the chemical potential, thereby leading to an average system density equal to the desired value $\rho_{\mathrm{eq}}=v_{\mathrm{eq}}^{-1}$. The relevant thermodynamic relationship is now

$$
\left.\frac{\partial(\mu)}{\partial v}\right|_{T}=\left.v \frac{\partial P}{\partial v}\right|_{T}
$$

which, to first order, becomes

$$
\mu_{\mathrm{eq}}=\mu_{0}+\left\langle\kappa_{T}^{-1}\right\rangle\left(v_{\mathrm{eq}}-v_{0}\right),
$$

where the brackets on the isothermal compressibility denote an average of the initial and final values when a trapezoidal rule is used; $\kappa_{T}$ can be evaluated from the fluctuations of the number of molecules in an (expanded) grand canonical simulation [see Eq. (16)]. The method then proceeds in a manner analogous to that outlined in Sec. IV for the implementation of pseudo-NPT simulations.

\section{GIBBS-DUHEM INTEGRATION METHODS}

\section{A. Pure component systems}

A pure component Gibbs-Duhem equation for phase "I" can be written as

$d(\beta \mu)^{\mathrm{I}}=h^{\mathrm{I}} d \beta^{\mathrm{I}}+v^{\mathrm{I}} \beta^{\mathrm{I}} d P^{\mathrm{I}}$, where $h^{\mathrm{I}}$ and $v^{\mathrm{I}}$ are the molar enthalpy and volume, respectively. For two phases I and II at coexistence, these equations can be solved in the $P-\beta$ plane to yield the Clapeyron equation,

$$
\left[\frac{d \ln P}{d \beta}\right]_{s}=-\frac{h^{\mathrm{I}}-h^{\mathrm{II}}}{\beta P\left(v^{\mathrm{I}}-v^{\mathrm{II}}\right)},
$$

where the subscript $s$ indicates that the derivative is taken along the saturation line. Kofke ${ }^{5}$ showed that numerical integration of Eq. (19) can be accomplished provided that an initial condition (i.e., a coexistence point) is known. The right-hand side of Eq. (19) must be evaluated through simultaneous NPT simulations of both phases. Details of the technique can be found in the original reference.

Kofke's implementation is the natural choice when it is important to avoid simulations involving particle insertions in favor of constant pressure simulations. The problem is now reformulated based on the opposite strategy. Writing Eq. (18) for phases I and II and recognizing that at coexistence $d P^{\mathrm{I}}=d P^{\mathrm{II}}$ and $d(\beta \mu)^{\mathrm{I}}=d(\beta \mu)^{\mathrm{II}}$, we can eliminate $d P$ from both equations to arrive at

$$
\left[\frac{d(\beta \mu)}{d \beta}\right]_{s}=\frac{\rho^{\mathrm{II}} h^{\mathrm{II}}-\rho^{\mathrm{I}} h^{\mathrm{I}}}{\rho^{\mathrm{II}}-\rho^{\mathrm{I}}}=\frac{\rho^{\mathrm{II}} u^{\mathrm{II}}-\rho^{\mathrm{I}} u^{\mathrm{I}}}{\rho^{\mathrm{II}}-\rho^{\mathrm{I}}},
$$

where $\rho$ is the molar (or number) density of the phase. $\mathrm{Nu}$ merical integration of this equation also requires an initial coexistence point but, in contrast to the case of Clapeyron's equation, it now entails simultaneous grand canonical simulations of both phases (volume moves are not required). For polymers, an expanded GC approach is of course the method of choice (see the discussion pertaining to Fig. 1).

To demonstrate the validity of the method suggested by Eq. (20), we follow Kofke $\mathrm{K}^{5}$ and apply it to the van der Waals fluid, the compressibility factor is given by

$$
Z=\frac{1}{1-4 \eta}-\frac{27}{2} \eta \beta_{r},
$$

where $\beta_{r}$ is the reciprocal temperature divided by its value at the critical point. Since absolute values of the molar enthalpy are required in Eq. (20), care must be exercised in employing consistent equations for $\mu$ and $h$. The chemical potential is (within an additive constant)

$$
\beta \mu=\log \left(\beta_{r}^{3 / 2} \eta\right)+Z-1-\frac{27}{2} \eta \beta_{r}-\log (1-4 \eta) .
$$

The corresponding molar energy is given by

$$
\beta u=\frac{3}{2}-\frac{27}{2} \eta \beta_{r} .
$$

The difference of calculated pressures on each phase could provide a measure of the departure from coexistence. We integrated Eq. (20) from $\beta_{r}=2$ to $\beta_{r}=1.1$ using a simple trapezoid predictor-corrector method. Note that once a new estimate of $\beta \mu$ has been generated, the coexisting densities of each phase required to evaluate the right-hand side of Eq. (20) must be obtained from Eq. (22). As expected, the accuracy of the calculated values of $\beta \mu, P$, and coexistence densities all improve as the integration step size is made smaller. Figure 3 shows coexisting densities for two step sizes: 0.3 


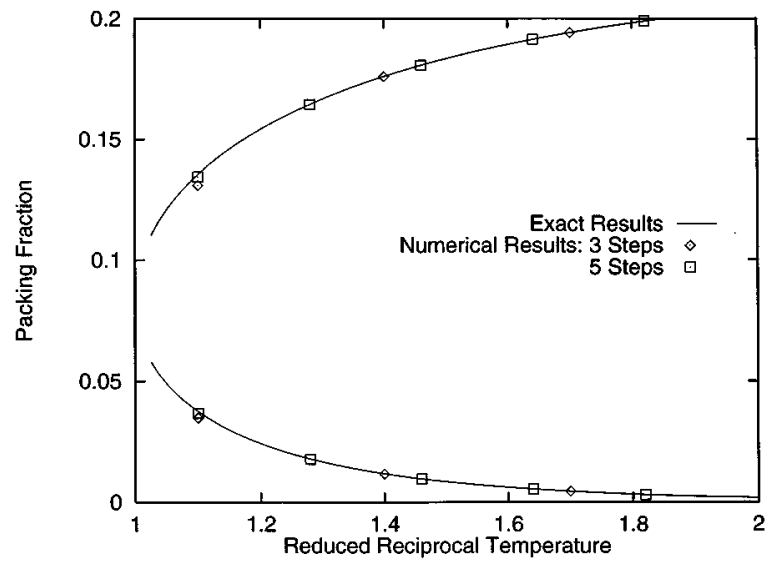

FIG. 3. Phase envelope of the van der Waals fluid calculated by numerical integration of Eq. (20) in the plane of packing fraction vs reduced reciprocal temperature $\left(\eta-\beta_{r}\right)$. Two sets of results are shown to illustrate the effect of integration step size on the accuracy of the process; in both cases the integration was started at $\beta_{r}=2.0$

(three steps) and 0.18 (five steps); the deviations from the exact results are only visible for the last points (at $\beta_{r}=1.1$ ). In practice, one should employ smaller step sizes as the critical region is approached.

Figure 4 shows a comparison of the right-hand sides of Eqs. (19) and (20) as a function of $\beta_{r}$. While the integrand of Eq. (19) varies more slowly than that of Eq. (20) (which is numerically favorable), the latter is sufficiently smooth to anticipate good numerical properties. In some cases, Eq. (20) could be rearranged to render a more insensitive integrand, for the example at hand, however, no simple change proved advantageous.

\section{B. Application to a single component system}

We studied the vapor-liquid coexistence of an octamer system whose sites interact through a square-well potential energy with a well width of $0.5 \sigma$. We employed the second-

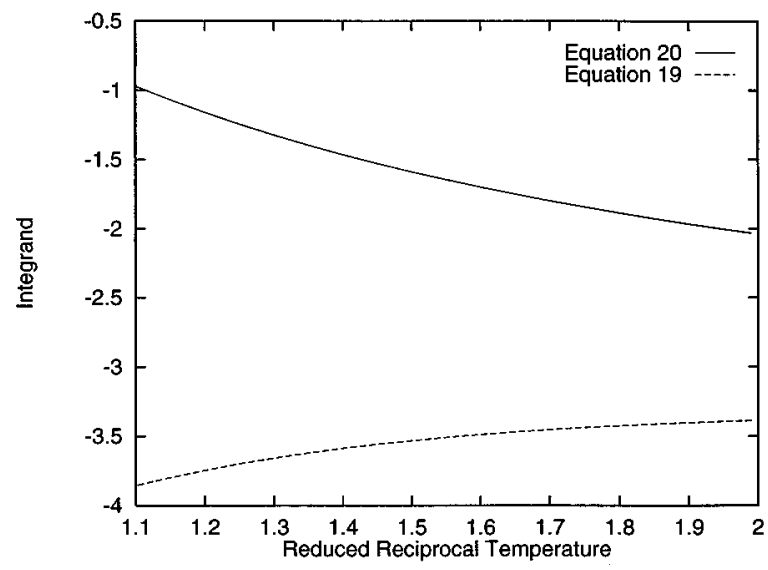

FIG. 4. Comparison of the integrands given by Eq. (19) (corresponding to the Clapeyron form of the Gibbs-Duhem equation) and Eq. (20) (corresponding to the form proposed in this work) as a function of reduced reciprocal temperature $\left(\beta_{r}\right)$.

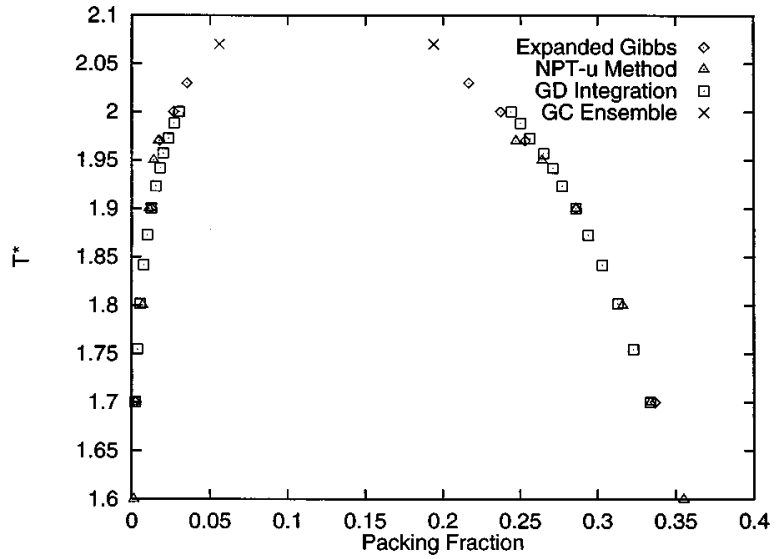

FIG. 5. Coexistence data for square-well octamers. The diamonds show results of expanded Gibbs simulations (Ref. 9), the triangles depict results of the NPT- $\mu$ method (Ref. 19), and the squares show results from the Gibbs-Duhem (GD) integration presented in this work (started at $T^{*}=1.9$ ). The coexistence point at $T^{*}=2.07$ was obtained by the grand canonicalhistogram reweighting technique (see the text).

order corrector-predictor formulas described in the Appendix. In all cases, the size of the simulation box was selected such that $100<N<150$ molecules were present in each simulation phase; the parameters for the expanded GC simulation were: $\Delta n_{y}=n_{y+1}-n_{y}=4, N_{\text {sp }}=20$, and $N_{\text {state }} \sim 0.5 N$. Simulations were started at the $T^{*}=1.9$ coexistence point; accurate data for that point were obtained from a long expanded Gibbs ensemble simulation. Gibbs-Duhem integrations were conducted by both increasing and decreasing the value for $\beta$. Our results are shown in Table I and Fig. 5. For comparison, we also show coexistence data obtained from the $N P T-\mu$ method, ${ }^{19}$ expanded Gibbs ensemble simulations, ${ }^{9}$ and from a combination of grand canonical and histogram reweighting techniques ${ }^{20}$ as described by Wilding $^{21}$ (for the highest temperature point at $T^{*}=2.07$ ). Good agreement is found between all sets of data. In particular, the results from our Gibbs-Duhem integration agree well (within the uncertainty of the simulations) with those obtained by other techniques; the agreement appears to be somewhat better for $T^{*}<1.9$ while systematic deviations are observed for $T^{*}>1.95$. These deviations are the result of an accumulation of errors from the integrator and from the simulation results (i.e., finite size effects, etc.), which are exacerbated as the critical region is approached. Some of the most immediate measures that could be used to alleviate this problem would be to employ a smaller step size, run longer simulations, and employ an improved integrator (for our purposes no such refinements seem warranted). The method will eventually fail (regardless of the integration step size) as the critical point is approached and phase reversal becomes prevalent (a shortcoming shared by the implementation in Ref. 8).

Although the coexistence envelope is commonly mapped from a low temperature point (high $\beta$ ) to a high temperature point (low $\beta$ ), the inverse route appears to be more suitable for polymer systems. Close to the critical point, a grand canonical simulation will easily sample states from both vapor 
and liquid phases (due to strong density fluctuations), thereby providing a route to calculate accurately coexistence points $^{21}$ (see the data point at $T^{*}=2.07$ in Fig. 5). Wilding has employed such a methodology and has also implemented a method that resorts to further iterative techniques in order to map the coexistence envelope at lower temperatures. The Gibbs-Duhem integration proposed here provides an alternative, more straightforward way of performing such a task. Further, since both approaches rely on grand canonical simulations, it should in principle be possible to formulate a procedure that combines their strengths. This and other applications will be reported in the future.

\section{Multicomponent systems}

Mehta and $\mathrm{Kofke}^{8}$ have presented extensions of the single-component Gibbs-Duhem integration technique to multicomponent systems. Again, their strategy consists of avoiding simulations entailing fluctuations of the number of particles whenever possible. For two-component systems, these authors proposed two forms of the Clapeyron equation which imply two different simulation methodologies: (i) a "semigrand" method, which requires simulations at constant $T, P$, total number of particles (in both phases) and fugacity fraction; the latter constraint entails moves that exchange the identity of the two species, and (ii) an "osmotic" method, which requires simulations at constant $T, P$, number of particles of one of the species (in each phase), and the chemical potential of the other species; the latter condition entails insertion or deletion attempts for the corresponding species.

Our alternative viewpoint favors constant- $\mu$ simulations for polymer systems and can be accommodated more naturally into the osmotic method than the semigrand alternative. For this purpose, an expanded grand canonical type of approach could be exploited if the constant- $\mu$ element of the osmotic "ensemble" was to be applied to a polymeric species. The semigrand approach could also be implemented in an expanded ensemble manner, provided both species bear enough similarities. We now propose a formulation more appropriate for polymer blends of arbitrary dissimilarity. For a two-component system, the Gibbs-Duhem equation can be written as

$$
x_{1} d\left(\beta \mu_{1}\right)+x_{2} d\left(\beta \mu_{2}\right)=h d \beta+v \beta d P .
$$

For two coexisting phases I and II at equilibrium we have $d\left(\beta \mu_{i}\right)^{\mathrm{I}}=d\left(\beta \mu_{i}\right)^{\mathrm{II}}, d P^{\mathrm{I}}=d P^{\mathrm{II}}$, and $d \beta^{\mathrm{I}}=d \beta^{\mathrm{II}}$. Under these conditions, Eq. (24) for the two phases can be solved to yield

$$
\begin{aligned}
d \beta \mu= & \frac{\left[\left(1-x_{i}^{\mathrm{I}}\right) h^{\mathrm{II}}-\left(1-x_{i}^{\mathrm{II}}\right) h^{\mathrm{I}}\right]}{\left(x_{i}^{\mathrm{II}}-x_{i}^{\mathrm{I}}\right)} d \beta \\
& +\frac{\left[\left(1-x_{i}^{\mathrm{I}}\right) v^{\mathrm{II}}-\left(1-x_{i}^{\mathrm{II}}\right) v^{\mathrm{I}}\right]}{\left(x_{i}^{\mathrm{II}}-x_{i}^{\mathrm{I}}\right)} \beta d P, \quad i=1,2 .
\end{aligned}
$$

Depending on the working plane selected, we can generate isothermal diagrams $(d \beta=0)$ or isobaric diagrams $(d P$
$=0$ ): in either case the corresponding term in the right-hand side of Eq. (25) disappears, thereby resulting in a first-order differential equation.

Starting from a known coexistence point, integration of Eq. (25) can be accomplished by predictor-corrector techniques similar to those employed in Ref. 8 for either $d \beta=0$ or $d P=0$. Since implementations for the isothermal case have already been examined by Mehta and Kofke (i.e., the semigrand and osmotic methods), we focus our attention on the isobaric case for which Eq. (25) reduces to

$$
\left[\frac{d \beta \mu_{i}}{d \beta}\right]_{P, s}=\frac{\left[\left(1-x_{i}^{\mathrm{I}}\right) h^{\mathrm{II}}-\left(1-x_{i}^{\mathrm{II}}\right) h^{\mathrm{I}}\right]}{\left(x_{i}^{\mathrm{II}}-x_{i}^{\mathrm{I}}\right)} .
$$

Simulations on this plane can be performed in two ways:

(1) Osmotic method, similar in spirit to Mehta and Kofke's implementation. ${ }^{8}$ Simulations are performed at constant $T(\beta), P, N_{1}$, and $\mu_{2}$. This method is to be preferred if the components differ greatly in segment size (i.e., species 2 should be selected as the one with the smaller segments).

(2) $V T \mu_{1} \mu_{2}$ (or grand canonical type) method, simulations are performed at constant $T$ and chemical potential of the two species [as governed by Eq. (26)], and volume of the system (the required extensive variable).

The $V T \mu_{1} \mu_{2}$ method outlined above is of particular interest for polymeric systems because (i) volume moves are not required and, as previously discussed, they represent the most important bottleneck for simulation of large systems, and (ii) simulations can be performed in an expanded GC fashion.

Extensions of these ideas to multicomponent systems with more than two components or to multiphase systems with more than two phases can be easily proposed; the discussion of Mehta and Kofke of multicomponent systems is also relevant to our work. ${ }^{8}$ As the number of components increases, so do the number of degrees of freedom and the possible ways of formulating integration schemes for the Gibbs-Duhem equations. For example, in a three-component system, a $V T \mu_{1} \mu_{2} \mu_{3}$ method could be implemented provided the chemical potential of one of the components (besides $T$ and $P$ ) is specified (and kept fixed); variations of that theme may include a $V T \mu_{1} \mu_{2} x_{3}$ method, etc.

\section{Application to multicomponent systems}

The $V T \mu_{1} \mu_{2}$ method of the previous section was employed to generate liquid-liquid coexistence data for a blend of short chains. The test system consisted of a symmetric binary mixture of octamers where nonbonded sites interact through a square-well potential energy function with uniform hard-core diameter $\sigma_{1}=\sigma_{2}=\sigma$, well width $=0.5 \sigma$, and well depths $\epsilon_{1,1}=\epsilon_{2,2}, \epsilon_{1,2}=0.8 \epsilon_{1, \mathrm{i}}(i=1,2)$. The pressure of the system was set to $P^{*}=P \sigma^{3} / \epsilon_{1, i}=0.1$. The parameters of the expanded GC method were $\Delta n_{y}=4, N_{\mathrm{sp}}=18$, and $N_{\text {state }}$ $\sim 0.5 \mathrm{~N}$. Given the expected symmetry in the composition of the coexisting phases, it is possible to perform the Gibbs- 
Duhem integration by simulating only one phase; for the sake of generality, however, we conducted full two-phase simulations.

Reference results were obtained from expanded Gibbs ensemble simulations; ${ }^{9,22}$ conventional continuum configurational bias Gibbs ensemble simulations, which are also practical for these short chains, were also performed for select points. ${ }^{23}$ For both Gibbs ensemble and $V T \mu_{1} \mu_{2}$ ensemble simulations, the size of the system was chosen such that (i) at least a total of 250 molecules were present in each phase, and (ii) at least 25 molecules of the dilute component were present. For temperatures far from the critical value, these guidelines resulted in simulation phases containing well above 3000 sites.

The starting coexistence point for the Gibbs-Duhem integration was chosen at $T^{*}=2.1$. Repeated attempts to generate highly accurate results for such a point (by employing either expanded or conventional Gibbs ensemble simulations) were somewhat fruitless; density averages in the range of $0.245<\eta<0.255$ were accompanied by molar composition fluctuations in the range of $0.115>x_{1}>0.09$. Slow relaxation of large-amplitude fluctuations of the volume (at coexistence conditions) appears to be the main culprit here; this may also pose a formidable obstacle for Gibbs-Duhem integration schemes involving volume changes. Although a constant density Gibbs ensemble simulation could be performed for this system (given the symmetry of the mixture), the results of such a method have been shown to be suffer from significant finite-size effects ${ }^{24}$ and the desired pressure cannot be directly enforced. These observations lend support to the view that the Gibbs ensemble methodology, while extremely practical for quickly drafting coexistence envelopes for chain systems, is not particularly well suited for generating results of high accuracy.

Since the success of the Gibbs-Duhem integration approach greatly depends on the accuracy of the initial coexistence point, there is a clear need for methods capable of providing data of high quality. For the purposes of this study, however, we had to settle for our best estimate of the $T^{*}=2.1$ coexistence point from continuum configurational bias Gibbs ensemble simulations. Our results for the isobaric coexistence curve are presented in Fig. 6 and Table II. Satisfactory agreement is found between the simulated data from Gibbs-Duhem integration (with the predictorcorrector scheme given in the Appendix) and that from the expanded Gibbs ensemble method. While both sets of data are statistically indistinguishable for $T^{*}<2.10$, a systematic deviation is observed for $T^{*}=2.15$. In addition to the admittedly low-accuracy initial point and to relatively large step sizes, the detrimental effects of large statistical noise also come into play as the temperature is increased.

A delicate problem observed in $V T \mu_{1} \mu_{2}$ simulations is the relative ease with which spontaneous (unilateral) phase reversal can occur (particularly at high $T^{*}$ ), thereby invalidating the results. Although the proclivity to such large fluctuations in composition is highly system dependent (e.g., it is significant in our system due to the small dissimilarity between the two species) some corrective measures must be

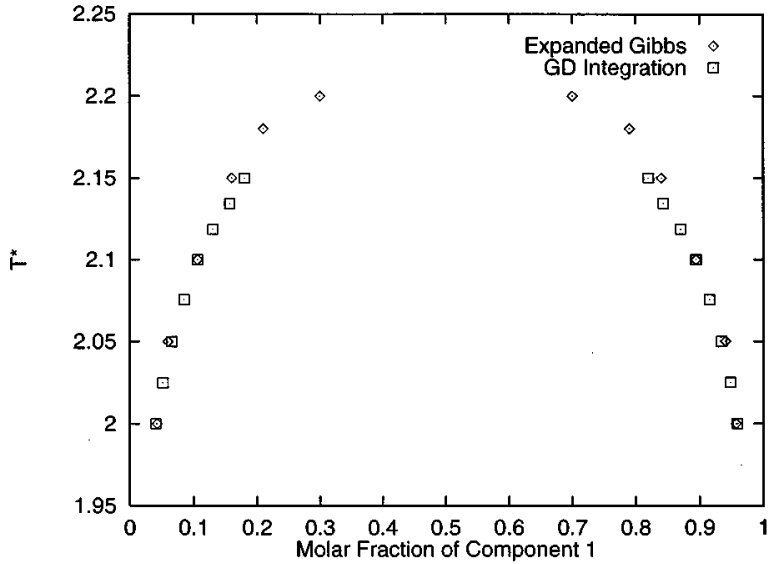

FIG. 6. Liquid-liquid binodal coexistence curve for a symmetric, binary blend of octamers at a pressure of $P^{*}=0.1$. Nonbonded chain sites interact through a square-well potential energy function with uniform hard-core diameter $(\sigma)$ and well width $=0.5 \sigma$; the cross interaction parameter is $\epsilon_{1,2}=0.8 \epsilon_{i, i}$ and $\epsilon_{1,1}=\epsilon_{2,2}$. Results are presented for expanded Gibbs simulations (diamonds) and for the Gibbs-Duhem, $V T \mu_{1} \mu_{2}$ integration method (squares) started at the $T^{*}=2.1$ point.

taken to prevent early failure of the method. In our case, it proved effective to increase the size of the system and to tune the relative frequency of transition attempts of both species. As for the pure component methodology proposed in Sec. VI A, the problem of phase reversal could also be (partially) alleviated by coupling the fluctuations in the number of molecules between the two phases [in a process reminiscent of (partial) particle transfer moves in Gibbs-type ensembles]. This coupling is detrimental to algorithmic simplicity, flexibility, and efficiency of the method; nevertheless, such a strategy is likely to be necessary if results are to be obtained in the vicinity of the critical point. For integration of the Clapeyron equation, ${ }^{5}$ coupling of volume fluctuations was found effective in delaying the onset of phase reversal.

TABLE II. Results of (expanded) Gibbs ensemble simulations and of the $V T \mu_{1} \mu_{2}$ Gibbs-Duhem integrations for the phase coexistence in a symmetric mixture of square-well octamers with $\epsilon_{1,2}=0.8 \epsilon_{1,1}=0.8 \epsilon_{2,2}$ and $\sigma_{1,2}=\sigma_{1,1}$ $=\sigma_{2,2}$ at $P^{*}=0.1$. The Gibbs-Duhem integrations were started at the $T^{*}=2.1$ point. The values reported for $x_{\text {dilute }}$ correspond to the average molar fraction of the dilute component in both phases.

\begin{tabular}{ccccccc}
\hline \hline & & \multicolumn{2}{c}{ (Expanded) Gibbs } & & \multicolumn{2}{c}{$V T \mu_{1} \mu_{2}$ method } \\
\cline { 3 - 4 } \cline { 6 - 7 }$\beta$ & $T^{*}$ & $\eta$ & $x_{\text {dilute }}$ & & $\eta$ & $x_{\text {dilute }}$ \\
\hline 0.5000 & 2.0000 & $0.278(7)$ & $0.043(8)$ & & 0.281 & 0.041 \\
0.4938 & 2.0251 & $\ldots$ & $\ldots$ & & 0.274 & 0.052 \\
0.4878 & 2.0500 & $0.268(5)$ & $0.060(7)$ & & 0.266 & 0.066 \\
0.4818 & 2.0756 & $\ldots$ & $\ldots$ & & 0.257 & 0.084 \\
0.4762 & 2.1000 & $0.249(4)$ & $0.105(8)$ & & \\
0.4720 & 2.1186 & $\ldots$ & $\ldots$ & & 0.241 & 0.130 \\
0.4685 & 2.1345 & $\ldots$ & $\ldots$ & & 0.234 & 0.157 \\
0.4651 & 2.1500 & $0.238(5)$ & $0.160(8)$ & & 0.230 & 0.180 \\
0.4587 & 2.1800 & $0.227(5)$ & $0.210(9)$ & & $\cdots$ & $\cdots$ \\
0.4545 & 2.2000 & $0.215(7)$ & $0.300(20)$ & $\cdots$ & $\cdots$ \\
\hline \hline
\end{tabular}




\section{CONCLUDING REMARKS}

A counterintuitive approach to the formulation of pseudo-ensemble simulations and Gibbs-Duhem integration schemes has been pursued based on the premise that fluctuations in the number of particles of polymeric species can be effectively accomplished by means of expanded-ensemble moves.

A pseudo- $N P T$ simulation method has been implemented in which volume fluctuations are replaced by expanded GC moves. This method has been shown to be more efficient than existing constant pressure algorithms for large systems of dense athermal homopolymers. Furthermore, the method could offer important advantages for isobaric simulations in parallel computer architectures. Extensions of pseudoensemble simulations to other ensembles have also been discussed. A sample implementation of pseudo-Gibbs simulations has been applied to determine a vapor-liquid coexistence point for square-well octamers.

Simulations to integrate numerically the Gibbs-Duhem equation have also been formulated; these exploit (expanded) grand canonical moves and forsake the need for volume fluctuations. For pure fluids and multicomponent systems, coexistence envelopes can be mapped starting from a known coexistence point. Our prescriptions for Gibbs-Duhem integrations have been tested by generating portions of the vapor-liquid envelope of square-well octamers and of the liquid-liquid coexistence curve of a symmetric binary blend of square-well octamers (with dissimilar cross interaction parameter). These systems were selected on the grounds of simplicity; it is hoped that these methods will prove advantageous for study of more complex systems.

The need for simulation methods that can provide the initial coexistence point for Gibbs-Duhem integrations with high accuracy has been emphasized; this can be viewed as one of the main limitations for the applicability of GibbsDuhem techniques. Alternative methods to the Gibbs ensemble appear to be in order (at least for some polymeric systems). Examples of such methods are the grand canonical-histogram reweighting approach, ${ }^{19}$ the NPT $\mu$ method, ${ }^{25}$ thermodynamic scaling, ${ }^{26}$ thermodynamic integration, etc. Another interesting possibility involves the use of Gibbs-Duhem integrations from a state(s) where accurate data are more easily attainable; for instance, starting from a coexistence point of a short chain, one could conduct an integration over chain length (following a strategy similar to that proposed in Ref. 27) until reaching the chain length of interest.

There is, of course, a limiting density above which grand canonical-type simulations are no longer practical. Although the segmental insertions required by the expanded ensembles could be aided by semianalytical mapping ${ }^{28}$ and gradual coupling, ${ }^{29}$ there are instances where volume fluctuations are to be preferred. For example, in dense systems where species differ greatly in segment size, it is advisable to avoid insertion or exchange moves of the large-segment component. We are continuing our investigation of vapor-liquid equilibrium, liquid-liquid equilibria, and phase transitions comprising or- dered phases for polymer blends of varying degrees of complexity. The methods proposed here are expected to facilitate the simulation of properties of such systems in the bulk and in constrained environments.

\section{ACKNOWLEDGMENTS}

This work was supported by a NYI grant from the National Science Foundation to J.J.dP. Additional support from the Donors of the Petroleum Research Fund, administered by the American Chemical Society, is gratefully acknowledged.

\section{APPENDIX}

Predictor-corrector schemes of order three or higher have been favored in previous implementations of GibbsDuhem integrations. ${ }^{5,8}$ Such schemes, however, have some disadvantages such as (i) different formulas must be employed for several steps at the start of the process, (ii) it is difficult to change the step size of integration, and (iii) they tend to be less stable than lower order schemes. Although higher order integrators can lead to more accurate results, it is the uncertainty in the simulated variables which will in all likelihood determine the overall accuracy of a Gibbs-Duhem integration scheme. Here we describe the second-order corrector schemes that were used in the applications presented in Secs. VI B and VI D; the predictor step for these applications is of little relevance and a simple first-order Euler formula was used. The formulas presented below provide a compromise between the simplest trapezoidal rule and higher order schemes, and have the advantage of facilitating changes in the integration step size which enhance flexibility and effectiveness. Two variants are presented which can be derived by combining suitably truncated Taylor series expansions of the dependent variable " $y$ " in terms of the independent variable " $x$ " (see, e.g., Ref. 30); their general form can be written as

$$
y_{k+1}=A y_{k-1}+B y_{k}+D \sum_{i=-1}^{+1} C_{i} y_{k+i}^{\prime} .
$$

For the first alternative we have

$$
\begin{aligned}
& A=0, \quad B=1, \\
& C_{-1}=-r^{-1}, \\
& C_{0}=r^{-1}+4+3 r, \\
& C_{1}=2+3 r, \\
& D=\frac{h_{k}}{6(1+r)} .
\end{aligned}
$$

For the second variant we have

$$
\begin{aligned}
& A=\frac{r^{-2}}{3+2 r}, \\
& B=1-\frac{r^{-2}}{3+2 r}, \\
& C_{-1}=0,
\end{aligned}
$$




$$
\begin{aligned}
& C_{0}=r^{-1}+2+r, \\
& C_{1}=1+r, \\
& D=\frac{h_{k}}{3+2 r} .
\end{aligned}
$$

In either case $r=h_{k-1} / h_{k}$ where $h$ is the integration step size, i.e., $h_{j}=x_{j+1}-x_{j}$.

These two variants gave almost identical results for the cases studied in this work.

\footnotetext{
${ }^{1}$ M. P. Allen and D. J. Tildesley, Computer Simulation of Liquids (Clarendon, Oxford, 1987).

${ }^{2}$ A. Z. Panagiotopoulos, Mol. Phys. 61, 813 (1987).

${ }^{3}$ J. J. de Pablo, M. Laso, and U. W. Suter, J. Chem. Phys. 96, 6157 (1992); D. Frenkel and B. Smit, Mol. Phys. 75, 983 (1992).

${ }^{4}$ S. K. Kumar, I. Szleifer, and A. Z. Panagiotopoulos, Phys. Rev. Lett. 66, 2935 (1991).

${ }^{5}$ D. A. Kofke, J. Chem. Phys. 98, 4149 (1993).

${ }^{6}$ M. Mehta and D. A. Kofke, Mol. Phys. 86, 139 (1995).

${ }^{7}$ P. J. Camp and M. P. Allen, Mol. Phys. 88, 1459 (1996).

${ }^{8}$ M. Mehta and D. A. Kofke, Chem. Eng. Sci. 49, 2633 (1994).

${ }^{9}$ F. A. Escobedo and J. J. de Pablo, J. Chem. Phys. 105, 4391 (1996).

${ }^{10}$ F. A. Escobedo and J. J. de Pablo, Macromol. Theory Simul. 4, 691 (1995).

${ }^{11}$ W. W. Wood, J. Chem. Phys. 48, 415 (1968).
}

${ }^{12}$ F. A. Escobedo and J. J. de Pablo, J. Chem. Phys. 102, 2636 (1995).

${ }^{13}$ J. Chang and S. I. Sandler, J. Chem. Phys. 103, 3196 (1995).

${ }^{14}$ F. A. Escobedo and J. J. de Pablo, J. Chem. Phys. 103, 1946 (1995).

${ }^{15}$ F. A. Escobedo and J. J. de Pablo, J. Chem. Phys. 103, 2703 (1995).

${ }^{16}$ F. W. Tavares, J. Chang, and S. I. Sandler, Mol. Phys. 86, 1451 (1995).

${ }^{17}$ A. Z. Panagiotopoulos, N. Quirke, M. Stapleton, and D. J. Tildesley, Mol. Phys. 63, 527 (1988).

${ }^{18}$ D. Moller and J. Fischer, Mol. Phys. 69, 463 (1990).

${ }^{19}$ F. Escobedo and J. J. de Pablo, Mol. Phys. 87, 347 (1996).

${ }^{20}$ A. M. Ferrenberg and R. H. Swendsen, Phys. Rev. Lett. 61, 2635 (1988).

${ }^{21}$ N. B. Wilding, Phys. Rev. E 52, 602 (1995); J. Chem. Phys. 105, 802 (1996).

${ }^{22}$ In Ref. 9 expanded Gibbs simulations are implemented for pure component systems; the extension to multicomponent systems is straightforward and follows similar guidelines as for conventional Gibbs ensemble in Ref. 17.

${ }^{23}$ M. Laso, J. J. de Pablo, and U. W. Suter, J. Chem. Phys. 97, 2817 (1992). G. C. A. M. Mooij, D. Frenkel, and B. Smit, J. Phys. Cond. Mat. 4, L255 (1992).

${ }^{24}$ J. R. Recht and A. Z. Panagiotopoulos, Mol. Phys. 80, 843 (1993).

${ }^{25}$ J. Vrabec and J. Fischer, Mol. Phys. 85, 781 (1995).

${ }^{26}$ J. P. Valleau, J. Comput. Phys. 96, 193 (1991).

${ }^{27}$ P. Camp, C. P. Mason, M. P. Allen, A. A. Khare, and D. Kofke, J. Chem. Phys. 105, 2837 (1996).

${ }^{28}$ F. Escobedo and J. J. de Pablo, Mol. Phys. 89, 1733 (1996).

${ }^{29}$ R. D. Kaminsky, J. Chem. Phys. 101, 4986 (1994).

${ }^{30}$ F. A. Escobedo, M. S. thesis, University of Nebraska-Lincoln, July 1993; F. Escobedo and H. Viljoen, Ind. Eng. Chem. Res. 34, 794 (1995). 